\title{
Mixing costs and switch costs when switching stimulus dimensions in serial predictions
}

\author{
Andrea M. Philipp • Claudia Kalinich • \\ Iring Koch $\cdot$ Ricarda I. Schubotz
}

Received: 30 March 2005 / Accepted: 4 June 2007 / Published online: 29 April 2008

(C) The Author(s) 2008

\begin{abstract}
Mixing costs and switch costs are two markers for the costs that arise in multitasking situations. To further explore mixing costs and switch costs, we used a serial prediction task in which subjects switched between stimulus dimensions (i.e., color, form, and position). Using this task, we demonstrate that both mixing costs and switch costs are influenced by task conflict and the resolution of interference. Here, we show that both mixing costs and switch costs are affected by a local factor, namely the necessity to resolve interference in the current trial in mixed blocks. However, whereas mixing costs can be sufficiently explained by interference resolution in the current trial, switch costs are also affected by carry-over effects from the preceding trial. As regards these carry-over effects, the present paradigm enabled us to demonstrate the influence of both persisting activation and persisting inhibition on the performance in switch trials.
\end{abstract}

\section{Introduction}

There is an amazing flexibility in human behavior. People are able to flexibly switch from one task to another and to keep in mind different goals. However, as we sometimes

A. M. Philipp ( $\square) \cdot$ I. Koch

Department of Psychology, RWTH Aachen University,

Jägerstrasse 17-19, 52066 Aachen, Germany

e-mail:philipp@psych.rwth-aachen.de

A. M. Philipp · I. Koch

Department of Psychology, Max Planck Institute for Human

Cognitive and Brain Sciences, Leipzig, Germany

C. Kalinich · R. I. Schubotz

Department of Cognitive Neurology, Max Planck Institute

for Human Cognitive and Brain Sciences, Leipzig, Germany realize in our daily life, there are also costs associated with such flexible behavior. The aim of the present study was to examine flexible, multitasking behavior and the costs that are associated with it.

We applied the methodology of a task-switching paradigm (for a review see Monsell, 2003) to explore multitasking behavior. More precisely, subjects switched their attention between stimulus dimensions in a serial prediction task (SPT, Schubotz, 1999). In our SPT, a repetitive sequence concerning the stimulus dimension color, form, or position was presented. This sequence consisted of three elements: "abc". For example, in a color sequence this could be red, blue, and green. The sequence was presented twice and subjects were asked to detect whether the repetitive stimulus sequence contained an unexpected deviance, that is, whether there was a complete repetition of the first three elements or whether the sequence was changed. In a deviant sequence, the last two elements were flipped (i.e., abcacb instead of abcabc). In the context of the SPT, a trial was defined as the presentation of an entire stimulus sequence comprising six elements. Therefore, in contrast to previous task-switching studies, we focused on a serial prediction based on a sequence of stimuli rather than a choice response decision based on a single stimulus (e.g., "Is the stimulus red or blue"). Yet, although the task requires a complex serial prediction, response requirements are actually minimal and do not differ among the stimulus dimensions. Using an SPT, we intended to increase the generality of previous studies by using a rather different kind of task. Note that effects in the SPT are measured primarily in the error data so that we focused on error data instead of RT data.

In task switching, switching between stimulus dimensions has been shown to result in costs (see, e.g., Meiran \& Marciano, 2002; Steinhauser \& Hübner, 2005). In general, 
two different kinds of costs can be observed. On the one hand, there are so-called "mixing costs" (Los, 1996; Meiran, 2000). Mixing costs describe the difference between performance in a situation in which only one stimulus dimension is relevant (i.e., "pure blocks") and situations in which people switch stimulus dimensions in "mixed blocks". On the other hand, within mixed blocks, it can be observed that performance is worse after a switch of the relevant stimulus dimension as compared to a repetition of the relevant stimulus dimension. This kind of cost is usually termed "switch costs" (Allport, Styles, \& Hsieh, 1994; Rogers \& Monsell, 1995) and is calculated as the performance difference between switch trials and repeat trials in mixed blocks. Mixing costs are either calculated as the general performance difference between pure and mixed blocks (see Koch, Prinz, \& Allport, 2005; Los, 1996) or as the difference between pure blocks and repetition trials of mixed blocks (see Kray \& Lindenberger, 2000; Meiran, 2000; Rubin \& Meiran, 2005). When calculated in the latter way, mixing costs can be cleanly separated from switch costs. Therefore, we used the latter definition of mixing costs in the present study.

Although both mixing costs and switch costs can be seen as indicating costs of multitasking, a number of differences between mixing costs and switch costs have been established in the recent literature. One of the first dissociations relates to the influence of aging. Whereas switch costs are comparable for young and older subjects, older adults show larger mixing costs than young adults (Kray \& Lindenberger, 2000; Mayr, 2001). Mixing costs and switch costs also show different result patterns in the comparison of children and young adults (Davidson, Amso, Anderson, \& Diamond, 2006). Additionally, mixing costs and switch costs were distinguished with respect to neural correlates in both an fMRI study (Braver, Reynolds, \& Donaldson, 2003) and an ERP study (Goffaux, Phillips, Sinai, \& Pushkar, 2006). These results suggest that mixing costs and switch costs depend on different neural networks and/or relate to different task processing stages. Consequently, mixing costs and switch costs are usually attributed to different origins.

One possibility to account for switch costs is to relate it to interference resolution and subsequent memory-based carry-over effects (see, e.g., Allport \& Wylie, 1999, for a review). In contrast, for mixing costs it is assumed that they do not represent such transient, trial-to-trial carry-over effects but more general, sustained effects (see, e.g., Los, 1996; Rubin \& Meiran, 2005). At first, such general effects were attributed to a higher memory load in mixed blocks because two tasks have to be kept active in working memory. Importantly, however, Rubin and Meiran (2005) showed that mixing costs cannot be explained by simply assuming a higher memory load in mixed blocks. Rather, mixing costs seem to arise as a consequence of task conflict. Specifically, mixing costs were observed for bivalent stimuli (i.e., stimuli that allow both tasks to be performed) but not for univalent stimuli (i.e., stimuli that allow only one task to be performed). Similarly, Koch et al. (2005) were able to demonstrate that mixing costs were higher for items that were mapped to two different tasks as compared to items that were mapped to one task only. Yet, whereas switch costs were not affected by the manipulation reported in the study of Koch et al. (2005), it is usually observed that switch costs are higher for bivalent than for univalent stimuli (e.g., Meiran, 2000). Thus, task conflict seems to affect both mixing costs and switch costs.

The aim of the present study was to demonstrate that, apart from all differences, both mixing costs and switch costs are affected by task conflict. In contrast to previous studies, however, we examined the influence of task conflict for both mixing costs and switch costs within the same experiment. We believe that both mixing costs and switch costs depend largely on the interference caused by a bivalent stimulus in a specific trial and that both kinds of costs are increased in situations in which interference has to be resolved. However, we also assume that partly different mechanisms account for mixing costs and switch costs. In particular, we hypothesize that mixing costs are mainly due to interference in the current trial (cf. Rubin \& Meiran, 2005), whereas switch costs additionally depend on carryover effects from the preceding trial (cf. Allport \& Wylie, 1999).

To explore the role of interference on both mixing costs and switch costs, we used multi-dimensional stimuli. The relevant stimulus dimension for the SPT could be color, form, or position. In each trial, the stimuli varied along two of these dimensions, whereas the third dimension was fixed. As only two of the three dimensions were variable in each trial, we consider the stimuli to be "two-dimensional". One of the variable dimensions was indicated as relevant dimension and had to be attended to by the subjects ("primary sequence"). The other variable dimension was not task-relevant and had to be ignored by the subjects ("secondary sequence"). Both sequences could be either deviant or non-deviant. However, subjects were instructed to respond only to the deviance of the primary sequence.

For the measurement of mixing costs and switch costs, we used both pure blocks and mixed blocks (see Table 1 for an overview of blocks). In pure blocks, subjects had to attend to the same primary stimulus dimension (e.g., color) in each trial. The secondary sequence could be one of the two remaining dimensions (i.e., form or position), whereas the last dimension was fixed. Thus, in each pure block, two different types of trials were possible. For example, in a pure color block, the combinations of primary and secondary sequence could be color/form and color/position. In mixed blocks, in contrast, subjects switched between two 
Table 1 Possible combinations of primary and secondary sequences in different pure and mixed blocks

\begin{tabular}{|c|c|c|c|}
\hline & \multicolumn{3}{|c|}{ Primary stimulus dimension } \\
\hline & Color & Form & Position \\
\hline \multicolumn{4}{|c|}{ Pure blocks ( 2 combinations of primary and secondary sequence in each block) } \\
\hline Color block & $\begin{array}{l}\text { Color/form } \\
\text { Color/position }\end{array}$ & & \\
\hline Form block & & $\begin{array}{l}\text { Form/color } \\
\text { Form/position }\end{array}$ & \\
\hline Position block & & & $\begin{array}{l}\text { Position/color } \\
\text { Position/form }\end{array}$ \\
\hline \multicolumn{4}{|c|}{ Mixed blocks ( 4 combinations of primary and secondary sequence in each block) } \\
\hline Color-form block & $\begin{array}{l}\text { Color/form (R) } \\
\text { Color/position (I) }\end{array}$ & $\begin{array}{l}\text { Form/color (R) } \\
\text { Form/position (I) }\end{array}$ & \\
\hline Color-position block & $\begin{array}{l}\text { Color/form (I) } \\
\text { Color/position (R) }\end{array}$ & & $\begin{array}{l}\text { Position/color (R) } \\
\text { Position/form (I) }\end{array}$ \\
\hline Form-position block & & $\begin{array}{l}\text { Form/color }(\mathrm{I}) \\
\text { Form/position (R) }\end{array}$ & $\begin{array}{l}\text { Position/color (I) } \\
\text { Position/form (R) }\end{array}$ \\
\hline
\end{tabular}

The first dimension always represents the primary sequence and the second dimension the secondary sequence. In mixed blocks, the term "(R)" following a sequence indicates a "relevant secondary sequence trial", that is, a trial in which the dimension of the secondary sequence could also be the primary sequence in the current block. The term "(I)" indicates an "irrelevant secondary sequence trial", that is, a trial in which the dimension of the secondary sequence never was the primary sequence in the current block

primary stimulus dimensions (e.g., color and form). For each possible primary sequence (e.g. color), the secondary sequence could be one of the two remaining dimensions (i.e., form or position). That is, in a color-form mixed block four different combinations of primary and secondary dimension could occur: (1) color/form, (2) color/position, (3) form/color, and (4) form/position (as primary/secondary sequence).

As a consequence of our design, two-dimensional stimuli were presented in each trial. Nevertheless, we would argue that, within these two-dimensional stimuli, we can differentiate between univalent and bivalent stimuli. This is because we consider two-dimensional stimuli as "functionally bivalent" only if the secondary sequence is a stimulus dimension that is currently activated in memory. That is, for each pure block, stimuli appear to be "univalent" because only one stimulus dimension is currently relevant and, thus, activated in memory. Consequently, in pure blocks no interference between potentially relevant stimulus dimensions should occur because only one dimension is relevant for sequence processing throughout the block (cf. Rubin \& Meiran, 2005). In contrast, in mixed blocks two different dimensions are potentially relevant and, thus, activated in working memory. Therefore, in a mixed block, interference might arise between the primary and the secondary sequence. However, interference should arise only in functionally bivalent trials in which the secondary sequence was potentially relevant in the present block. In the example of a color-form mixing block, color and form are potentially relevant. Thus, in the combinations color/ form and form/color the secondary sequence could also occur as relevant (i.e., primary) sequence in the block. Such trials are henceforth called "relevant secondary sequence trials". In contrast, no interference between primary and secondary sequence should occur in functionally univalent trials in which the dimension of the secondary sequence was never the primary sequence in the current block (in the example above: color/position and form/position because position is never the primary sequence in a color-form block; "irrelevant secondary sequence trials").

If task conflict and interference resolution in the current trial influence mixing costs, we predict that mixing costs should be observed when comparing the performance in pure blocks with that in relevant secondary sequence repeat trials in mixed blocks but not when comparing pure blocks with irrelevant secondary sequence repeat trials in mixed blocks. In other words, we assume that mixing costs occur because, for the same task, interference resolution is necessary in (a part of the trials in) mixed blocks but not in pure blocks.

Obviously, the same explanation cannot fully account for switch costs because relevant secondary sequence trials can be both task-repeat and task-switch trials. Thus, we do predict higher switch costs in relevant as compared to irrelevant secondary sequence trials. However, we believe that apart from the effect of interference resolution in the current trial, switch costs are also (and potentially to a larger degree) influenced by the after-effects of interference resolution in the preceding trial. That is, persisting activation of the relevant primary sequence and possibly persisting inhibition of the irrelevant secondary sequence from the last trial may affect performance in a current 
dimension-switch trial. Here, two different assumptions lead to our prediction that after-effects of interference resolution in the preceding trial affect the performance in switch trials and thus also increase switch costs.

On the one hand, if a stimulus dimension was relevant in the previous trial, it could be difficult to ignore or inhibit it because it is still activated as secondary sequence in the current trial (i.e., "competitor priming"). Here, we expected that persisting activation of the relevant dimension of the previous trial should lead to a reduced performance level in the current trial if this dimension becomes the secondary sequence. On the other hand, it could be more difficult to attend a primary sequence in the current trial that was irrelevant (and thus ignored or inhibited) in the previous trial (i.e., "negative priming", see Tipper 2001, for a review). Here, we expected that persisting inhibition has after-effects in the next trial such that it is more difficult to predict a sequence in a dimension that has been a secondary sequence in the preceding trial.

As our predictions are rather complex, we would like to illustrate them in examples (note that the examples always refer to a color-form block): The first example refers to the influence of task conflict on mixing costs. In a color-form mixed block, the two primary sequences can be color and form (cf. Table 1). Comparing the performance in irrelevant secondary sequence repeat trials (color/position and form/position) in these mixed blocks with the performance in pure blocks (i.e., the color block and the form block) should show similar error rates in both conditions, whereas mixing costs should occur when comparing the performance in relevant secondary sequence repeat trials (color/ form and form/color) with that in the corresponding pure blocks.

The second example refers to the prediction that the performance in switch trials is influenced by persisting activation and persisting inhibition. In each mixed block, eight different dimension-switch transitions are possible (cf. Table 2 for the possible transitions in a color-form block). Neither persisting activation nor persisting inhibition plays a role when subjects switch from a form/position trial to a color/position trial or vice versa ("baseline switch trials"). Both persisting activation and persisting inhibition can affect the performance when subjects switch from a form/color to a color/form trial or vice versa ("complete change switch trials"). Consequently, performance should be worse in the latter switch trials as compared to the former ones. To distinguish the influence of persisting activation and persisting inhibition, the remaining transitions are important. A previously relevant dimension that now became irrelevant might affect performance when subjects switch from a form/position to a color/form trial or from a color/position to a form/ color trial. These transitions are henceforth termed
Table 2 The different types of dimension-switch trials (i.e., baseline switch trials, complete change switch trials, attended $\operatorname{dn}_{(n-1)} \rightarrow \operatorname{ignored}_{(n)}$

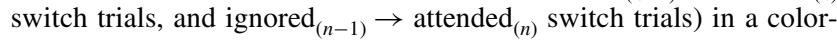
form mixing block as an example block

\begin{tabular}{llll}
\hline & Trial $n-1$ & & Trial $n$ \\
\hline Baseline & Color/position & $\rightarrow$ & Form/position \\
& Form/position & $\rightarrow$ & Color/position \\
Complete change & Color/form & $\rightarrow$ & Form/color \\
& Form/color & $\rightarrow$ & Color/form \\
Attended $_{(n-1)} \rightarrow$ ignored $_{(n)}$ & Color/position & $\rightarrow$ & Form/color \\
& Form/position & $\rightarrow$ & Color/form \\
$\operatorname{Ignored}_{(n-1)} \rightarrow \operatorname{attended~}_{(n)}$ & Color/form & $\rightarrow$ & Form/position \\
& Form/color & $\rightarrow$ & Color/position \\
\hline
\end{tabular}

"attended $d_{(n-1)} \rightarrow \operatorname{ignored}_{(n)}$ switch trials". A previously irrelevant dimension that became relevant in the current trial occurs when subjects switch from a color/form to a form/position trial or from a form/color to a color/ position trial. These transitions are henceforth termed "ignored ${ }_{(n-1)} \rightarrow \operatorname{attended}_{(n)}$ switch trials". The influence of persisting activation of a previously relevant dimension, thus, can be calculated by comparing the performance in baseline switch trials with that in attended $d_{(n-1)} \rightarrow \operatorname{ignored}_{(n)}$ switch trials; the influence of persisting inhibition can be calculated by comparing baseline switch trials and ignored $_{(n-1)} \rightarrow \operatorname{attended}_{(n)}$ switch trials. It is important to note that in this way, the present paradigm allows us to disentangle the influence of persisting activation and of persisting inhibition and to explore which of the two possible kinds of priming (i.e., competitor priming and negative priming) plays a role when switching between stimulus dimensions.

In summary, the present experiment examines cognitive flexibility and its costs by measuring mixing costs and switch costs when subjects switch attention between stimulus dimensions in a SPT. The main aim of the study was to demonstrate the influence of task conflict and interference resolution for both mixing costs and switch costs. The two main predictions were:

1. The hypothesis that mixing costs are caused by interference resolution in repeat trials of mixed blocks leads us to predict a lower error rate in pure blocks than in relevant secondary sequence repeat trials in mixed blocks (in which interference resolution is necessary). Yet, we also expect that there should be no difference between pure blocks and irrelevant secondary sequence repeat trials in mixed blocks (in which interference resolution is not necessary).

2. Apart from the influence of interference resolution in the current trial, the performance in switch trials and, thus, the size of switch costs should be influenced by carry-over effects from the preceding trial. Here, both 
persisting activation of a previously relevant sequence and persisting inhibition of a previously irrelevant sequence should increase the error rate in switch trials.

\section{Method}

Subjects

A total of twenty right-handed subjects (10 female and 10 male, mean age $=23.5$ years) were tested and received $17,50 €$ for their participation.

\section{Stimuli and tasks}

Subjects performed a SPT. Here, three successive stimuli formed a short sequence, which was repeated once (i.e., a total of six stimuli per trial). In half of the trials the repeated sequence was violated by shifting the positions of the last two stimuli of the trial (i.e., abcacb instead of abcabc for the primary sequence). Subjects had to report at the end of each trial whether the primary sequence ended unexpectedly, that is, with a deviance, or as predicted based on the first three stimuli.

Serial predictions were made with respect to the color, form, or position of a stimulus sequence. In each trial, stimuli were two-dimensional-that is, they had two different stimulus dimensions with a sequential structure. The third dimension was held constant. One stimulus dimension was relevant for the SPT (primary sequence), whereas the other dimension was not (secondary sequence). The primary sequence was presented as a sequence of three stimuli, whereas the secondary sequence was presented as an alternating sequence (i.e., ababab). Both the primary sequence and the secondary sequence could be either deviant or nondeviant. However, subjects were instructed to respond only according to the primary sequence.

The stimuli of each trial were presented one at a time. If the primary or the secondary sequence in a trial was based on the color of the stimuli, stimuli could be red, green, yellow, or blue. If color was neither the primary nor the secondary dimension, the color of the stimuli was gray. In the form dimension, stimuli were constructed by the outline form of a square (height $35 \mathrm{~mm} /$ width $33 \mathrm{~mm}$ ), a triangle (height $40 \mathrm{~mm}$ /width $35 \mathrm{~mm}$ ), a cross (height $34 \mathrm{~mm} /$ width $35 \mathrm{~mm}$ ), or a hexagon (height $37 \mathrm{~mm} /$ width $32 \mathrm{~mm}$ ). If form was held constant, a circle (diameter $33 \mathrm{~mm}$ ) was presented. The frame of each form was $6 \mathrm{~mm}$. For the position task the constant value was screen center. Otherwise, the stimuli could be shifted $3 \mathrm{~mm}$ upwards, to the left, downwards, or to the right. A central fixation sign was presented whenever position was the primary or secondary sequence to make position more salient. In each trial, three (for the primary sequence) or two (for the secondary sequence) out of the four possible dimension values were randomly selected with the constraint that two subsequent sequences were not identical.

At the beginning of each trial, the to-be-attended stimulus dimension was announced by the words "color", "form", or "position" (i.e., the German words "Farbe", "Form", and "Ort"). A cue was presented in each trial of the mixed blocks, but also in pure blocks. Subjects were not informed about the type of block (pure vs. mixed) or the potentially relevant stimulus dimensions within each block.

Responses were delivered by pressing one of two external keys, either with the right index finger, indicating a deviance in the sequence, or with the right middle finger, indicating that a sequence finished as expected. The external response keys measured $12 \mathrm{~mm} \times 17 \mathrm{~mm}$ and were separated by $10 \mathrm{~mm}$.

\section{Procedure}

The experiment was run in a single session of approximately $120 \mathrm{~min}$. After giving informed consent, subjects sat in a dimly lit, acoustically attenuated chamber in front of a 17" Trinitron Color Graphic Display (distance: $60 \mathrm{~cm}$ ). Subjects were informed about the SPT and the meaning of the response keys (i.e., deviant vs. non-deviant).

An experimental trial started with the visual cue presented for $500 \mathrm{~ms}$ in the screen center. Immediately afterwards the first stimulus was presented for $500 \mathrm{~ms}$, immediately followed by the next stimulus for $500 \mathrm{~ms}$, and so on. In practice trials in the beginning of the experiment stimuli were presented for $800 \mathrm{~ms}$ each, to allow subjects to adapt to the presentation speed. As a deviance of the sequence was detectable as soon as the fifth stimulus appeared, RT was measured from the onset of the fifth stimulus. Subjects had time to deliver their response from the onset of the fifth stimulus until $500 \mathrm{~ms}$ after the sixth stimulus had turned off (i.e., 1,500 ms). Subjects received a visual error feedback (i.e., a white " $\mathrm{X}$ " sign) for $350 \mathrm{~ms}$ when they pressed the wrong key (i.e., when they did not detect a deviance or erroneously reported a deviance) or when they responded too slowly (RT $>1,500 \mathrm{~ms}$ ). In trials with a correct response, the screen remained empty for $350 \mathrm{~ms}$. The next trial started $1,000 \mathrm{~ms}$ after the offset of the feedback (i.e., $1,850 \mathrm{~ms}$ after the offset of the sixth stimulus).

The experiment began with a practice part that included three short pure blocks (one block for each dimension) with four trials each and a mixed block of 36 trials (12 trials for each combination of two dimensions: color-form, colorposition, and form-position). The experiment itself consisted of nine blocks, with self-paced pauses between them. In three blocks, subjects had to attend to one stimulus 
dimension only (i.e., pure blocks; one for each dimension). In the remaining six blocks one of two possible dimensions had to be attended (i.e., mixed blocks; two for each combination of two stimulus dimensions). The two mixed blocks with the same dimension combination were always performed one after the other. Pure blocks and mixed blocks alternated in the experiment (i.e., one pure block-two mixed blocks). In each pure block, a stimulus dimension had to be attended that had not to be attended in the following two mixed blocks. Each block had 96 trials. However, each pure block and the first mixed block of each combination started with 16 practice trials, thereby increasing the number of trials to 112 altogether.

In a pure block, the primary sequence was the same in each trial (e.g., color). The secondary sequence in pure blocks could be either of the other two dimensions (e.g., form and position). The succession of trials was controlled for an equal number of each secondary dimension, repetition (vs. switch) of the secondary sequence, deviance of the primary sequence, deviance of the secondary sequence, and all combinations of these factors.

In mixed blocks, subjects switched between two primary sequences (e.g., color and form). Again, each primary sequence could be combined with either of the other two dimensions as secondary sequence. That is, a trial in which the color was the primary sequence could have either form or position as secondary sequence. Correspondingly, a trial in which the form was the primary sequence could have either color or position as secondary sequence. The succession of trials was controlled for an equal number of each dimension (primary as well as secondary), repetition (vs. switch) of the primary sequence, repetition (vs. switch) of the secondary sequence, deviance of the primary sequence, deviance of the secondary sequence, and all combinations of these factors.

\section{Data analysis}

The result section is divided into two parts. In a first step, we analyzed mixing costs and switch costs with respect to interference in the current trial. For mixing costs, relevance of the secondary sequence (pure block vs. relevant secondary sequence repeat trials in mixed blocks vs. irrelevant secondary sequence repeat trials in mixed blocks) and dimension (color vs. form vs. position) were used as within-subject independent variables. For switch costs, trial type (repeat trials in mixed blocks vs. switch trials in mixed blocks), relevance of the secondary sequence (relevant secondary sequence trials vs. irrelevant secondary sequence trials), and dimension (color vs. form vs. position) were used as within-subject independent variables. For variables with more than two levels, we report $\varepsilon$-values and use the Huynh-Feldt test to report $p$ values based on corrected degrees of freedom. However, we still report non-corrected degrees of freedom.

In a second step, we specifically examined switch trials in mixed blocks. Here, we tested whether switch costs are influenced by after-effects of the previous trial. In these analyses, we report theory-driven $t$ tests. In the first $t$ test, we compared baseline switch trials with complete change switch trials to see whether there is an effect at all. To differentiate between the influence of persisting activation and persisting inhibition, we compared the baseline switch trials with both attended $(n-1) \rightarrow$ ignored $_{(n)}$ switch trials and $\operatorname{ignored}_{(n-1)} \rightarrow \operatorname{attended}_{(n)}$ switch trials. Finally, we explored a possible interaction of persisting activation and persisting inhibition in an analysis of variance (ANOVA) including attended $d_{(n-1)} \rightarrow \operatorname{ignored}_{(n)}$ (present vs. not present) and ignored ${ }_{(n-1)} \rightarrow \operatorname{attended}_{(n)}$ (present vs. not present) as within-subject independent variables.

The dependent variable was the error percentage. As error data often violate the conditions for parametric tests, we tested the error distribution in each cell with a Kolmogorov-Smirnov test (error distributions showed no statistically significant deviation from a normal distribution, $Z s<1.24)$. Additionally, we also report non-parametric tests for the most important main effects. Significance was tested at $\alpha=0.05$.

\section{Results}

All practice trials and the first trial of each block were discarded from analysis. Only trials preceded by at least one correct trial were included in the data analysis.

Mixing costs, switch costs, and interference resolution in the current trial

For the analysis of mixing costs we conducted a $3 \times 3$ ANOVA with the within-subject variables relevance of the secondary sequence (pure block vs. relevant secondary sequence repeat trials in mixed blocks vs. irrelevant secondary sequence repeat trials in mixed blocks) and dimension (color vs. form vs. position). Error data are shown in Table 3.

The analysis revealed a significant main effect of relevance of the secondary sequence $(F(2,38)=5.1 ; \varepsilon=0.91$; $p<0.05)$. When tested in separate ANOVAs, the difference between pure blocks and irrelevant secondary sequence repeat trials was not significant $(F<1)$, whereas relevant secondary sequence repeat trials differed significantly from pure blocks $(F(2,38)=13.6 ; p<0.01)$ and irrelevant secondary sequence repeat trials $(F(2,38)=5.1 ; p<0.05)$. Thus, as predicted, the error rate in relevant secondary sequence repeat trials $(12.1 \%)$ was higher than in pure 
Table 3 Error percentage in pure blocks as a function of dimension (color vs. form vs. position) and error percentage in mixed blocks as a function of trial type (irrelevant secondary sequence repeat vs. relevant secondary sequence repeat vs. irrelevant secondary sequence switch vs. relevant secondary sequence switch) and dimension (color vs. form vs. position)

\begin{tabular}{lccc}
\hline & Color & Form & Position \\
\hline Pure blocks & 7.8 & 9.5 & 12.2 \\
Mixed blocks & & & \\
Irrelevant secondary sequence repeat & 7.6 & 10.9 & 11.5 \\
Relevant secondary sequence repeat & 10.0 & 14.0 & 12.4 \\
Irrelevant secondary sequence switch & 10.1 & 9.5 & 12.0 \\
Relevant secondary sequence switch & 12.6 & 16.6 & 16.0 \\
Mixing costs & & & -0.7 \\
Irrelevant secondary sequence repeat-pure & -0.2 & 1.4 & 0.2 \\
Relevant secondary sequence repeat-pure & 2.2 & 4.5 & 0.5 \\
Switch costs & & & \\
Irrelevant secondary sequence switch—irrelevant & 2.5 & -1.4 & 3.6 \\
$\quad$ secondary ssequence repeat & & & \\
Relevant secondary sequence switch-relevant & & & \\
$\quad$ secondary sequence repeat & 2.6 & & \\
\hline
\end{tabular}

blocks $(9.8 \%)$ and in irrelevant secondary sequence repeat trials $(10.0 \%)$. This result was also supported by non-parametric Wilcoxon tests $(Z<1$ for the comparison of pure blocks and irrelevant secondary sequence repeat trials in mixed blocks, $Z=3.2 ; N=20 ; p<0.01$ for the comparison of pure blocks and relevant secondary sequence repeat trials in mixed blocks, and $Z=2.1 ; N=20 ; p<0.05$ for the comparison relevant and irrelevant secondary sequence repeat trials in mixed blocks; averaged across stimulus dimensions).

In the $3 \times 3$ ANOVA, the effect of dimension was significant as well $(F(2,38)=4.0 ; \varepsilon=1.0 ; p<0.05)$. The data pattern shows $8.5 \%$ errors in color trials, $11.4 \%$ in form trials, and $12.1 \%$ in position trials. However, there was no significant interaction of relevance of the secondary sequence and dimension $(F<1)$.

For the analysis of switch costs, a three-way ANOVA was conducted with the within-subject variables trial type (repeat trials in mixed blocks vs. switch trials in mixed blocks), relevance of the secondary sequence (relevant secondary sequence trials vs. irrelevant secondary sequence trials), and dimension (color vs. form vs. position). This yielded a significant effect of trial type $(F(1,19)=8.2$; $p<0.01)$. Subjects had a lower error rate in dimension repetitions $(11.1 \%)$ than in dimension switches $(12.8 \%$, see Table 3), showing dimension-switch costs of $1.7 \%$. Again, the result was supported by a non-parametric Wilcoxon test $(Z=2.9 ; N=20 ; p<0.01$; averaged across stimulus dimension).

The main effect of dimension was close to significance $(F(2,38)=2.5 ; \varepsilon=1.0 ; p=0.092)$. The data pattern of the mixed blocks shows $10.1 \%$ errors in color trials, $12.8 \%$ in form trials, and $13.0 \%$ in position trials. Yet, the interaction of trial type and dimension was not significant $(F<1)$.
Further, the analysis yielded a significant main effect of relevance of the secondary sequence $(F(1,19)=18.1$; $p<0.001)$, indicating a higher error rate in relevant secondary sequence trials $(13.6 \%)$ than in irrelevant secondary sequence trials $(10.3 \%)$. Also, switch costs were numerically higher in relevant secondary sequence trials $(2.9 \%)$ than in irrelevant secondary sequence trials $(0.5 \%)$ but the corresponding interaction of trial type and relevance of the secondary sequence failed to reach significance $(F(1$, $19)=3.3 ; p=0.085)$. No other interaction was significant in the error analysis $(F \mathrm{~s}<1.9)$.

All in all, the first set of analyses showed that both mixing costs and switch costs can be found when subjects switch their attention between different stimulus dimensions in a SPT. Moreover, the results also provide clear evidence that interference in the current trial plays a crucial role. As regards mixing costs, the comparison of performance in pure blocks and relevant secondary sequence repeat trials in mixed blocks shows significant mixing costs of $2.3 \%$, whereas the comparison of the performance in pure blocks and irrelevant secondary sequence repeat trials in mixed blocks does not reveal clear mixing costs $(0.2 \%)$. Mixing costs, thus, were observed only in a condition in which the secondary sequence is potentially relevant and, thus, interference between the primary and secondary sequence has to be resolved in the repeat trials of the mixed block. With respect to switch costs, we could not demonstrate the same clear picture. Importantly, we found significant switch costs of $1.7 \%$. Although switch costs were numerically larger in relevant secondary sequence trials $(2.9 \%)$ than in irrelevant secondary sequence trials $(0.5 \%)$, this effect was not significant. However, it is important to note that the relatively small size of the switch costs might not have allowed an even more substantial and significant 
variation. Further, we assume that the performance in dimension-switch trials and consequently the size of switch costs is not only influenced by interference in the current trial but also by after-effects of the preceding trial. We explored the role of such after-effects in the second set of analyses.

After-effects of persisting activation and persisting inhibition

In the second set of analyses, we examined persisting activation and persisting inhibition of relevant and irrelevant stimulus dimensions on the performance in dimensionswitch trials in mixed blocks.

To analyze whether after-effects have an influence at all, we compared the error rate of baseline switch trials $(9.3 \%)$ with that of complete change switch trials $(16.7 \%$, see Table 4). The two-tailed $t$ test revealed a significant difference $(t(19)=4.7 ; p<0.001)$. The effect was replicated in the non-parametric Wilcoxon test $(Z=3.6 ; \quad N=20$; $p<0.001)$.

In order to differentiate between the consequences of persisting activation and persisting inhibition, we first compared baseline switch trials $(9.3 \%)$ with attended $(n-1) \rightarrow \operatorname{ignored}_{(n)}$ switch trials $(13.6 \%)$. The significant difference $(t(19)=3.0$; $p<0.01$; and $Z=2 ; N=20 ; p<0.05)$ indicates the occurrence of persisting activation. Further, we compared baseline switch trials $(9.3 \%)$ and ignored $_{(n-1)} \rightarrow$ attended $_{(n)}$ switch trials $(12.1 \%)$. Again, the difference was significant $(t(19)=2.3 ; p<0.05$; and $Z=2.1 ; p<0.05)$, indicating the occurrence of persisting inhibition.

To explore a possible interaction of persisting activation and persisting inhibition, we conducted a $2 \times 2$ ANOVA with the within-subject independent variables attended ${ }_{(n-1)}$

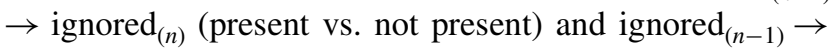
attended $_{(n)}$ (present vs. not present). The error analysis yielded significant main effects of attended $(n-1) \rightarrow$ ignored $_{(n)}$ $(F(1,19)=16.2 ; p<0.001)$ and $\operatorname{ignored}_{(n-1)} \rightarrow \operatorname{attended}_{(n)}$ $(F(1,19)=10.4 ; p<0.01)$. Importantly, the interaction of persisting activation and persisting inhibition was not significant $(F<1)$.

Taken together, the data pattern shows that subjects made more errors in both attended $_{(n-1)} \rightarrow$ ignored $_{(n)}$ switch trials and $\operatorname{ignored}_{(n-1)} \rightarrow \operatorname{attended}_{(n)}$ switch trials than in

Table 4 Error percentage in the different types of switch trials in mixed blocks

\begin{tabular}{lr}
\hline Baseline switch trials & 9.3 \\
Complete change switch trials & 16.7 \\
Attended $_{(n-1)} \rightarrow$ ignored $_{(n)}$ switch trials & 13.6 \\
Ignored $_{(n-1)} \rightarrow$ attended $_{(n)}$ switch trials & 12.1 \\
\hline
\end{tabular}

baseline switch trials. This finding indicates that both the persisting activation of a no longer relevant dimension and the persisting inhibition of a previously irrelevant dimension reduce task performance in switch trials. Further, the non-significant interaction of attended $\mathrm{d}_{(n-1)}$ $\rightarrow$ ignored $_{(n)}$ and $\operatorname{ignored}_{(n-1)} \rightarrow$ attended $_{(n)}$ suggests that effects of persisting activation and persisting inhibition are additive.

\section{Discussion}

Subjects in the present experiment flexibly switched between stimulus dimensions in a SPT. Although the results indicate that subjects were able to accomplish such flexible behavior, we also found costs. On the one hand, subjects showed more errors in mixed blocks as compared to pure blocks (i.e., mixing costs); on the other hand, the performance was worse in dimension-switch trials as compared to dimension-repeat trials (i.e., switch costs). In previous studies, it was often argued that mixing costs and switch costs have different characteristics and have to be attributed to different mechanisms (see, e.g., Kray \& Lindenberger, 2000; Braver et al., 2003). In contrast to these studies, we were able to demonstrate that both mixing costs and switch costs are influenced by task conflict and interference resolution (cf. Rubin \& Meiran, 2005).

Furthermore, the present study provides important new insights into the mechanisms underlying mixing costs and switch costs. As regards mixing costs, we observed that the error rate in relevant secondary sequence repeat trials was higher than in irrelevant secondary sequence repeat trials. In other words, dimension-repeat trials showed a higher error rate when the stimulus in the current trial was (functionally) bivalent than when it was (functionally) univalent (cf. Mayr, 2001; Mayr, Diedrichsen, Ivry, \& Keele, 2006). Consequently, we were able to demonstrate that mixing costs can be observed in the comparison of pure blocks and relevant secondary sequence trials, that is, trials in which the dimension of the secondary sequence is potentially relevant in the current mixed block. In contrast, we found no mixing costs when we compared pure blocks with irrelevant secondary sequence repeat trials in mixed blocks. On the one hand, this indicates that task conflict and the necessity to resolve interference between potentially relevant stimulus dimensions in mixed blocks leads to mixing costs. On the other hand, the data pattern also suggests that mixing costs do not (only) arise because a general default mechanism comes into play as soon as two dimensions are relevant in a given block. Rubin and Meiran (2005) demonstrated already that mixing costs depend on task conflict. In their study, mixing costs were observed only when stimuli had features of both relevant tasks. 
However, the comparison between such bivalent and univalent stimuli was one between subjects. Therefore, the present study does not only replicate the role of task conflict but extends it in an important aspect. We were able to demonstrate that mixing costs are due to the resolution of a local interference when both potentially relevant dimensions appear in any given trial. This shows that mixing costs are not a general finding when one compares the performance in pure and mixed blocks. Rather, the present results indicate that the local interference of each trial can affect the "global" mixing costs. Therefore, one should be cautious to differentiate between mixing costs and switch costs with respect to global vs. local costs only. This is especially important because it was also shown that "local" switch costs are affected by global features of the current task demands (cf. Kleinsorge, Heuer, \& Schmidtke, 2004).

With respect to switch costs, the experiment shows a numerical albeit not significant effect of interference in the current trial. This finding corresponds to previous studies reporting that switch costs were larger with bivalent than with univalent stimuli (see, e.g., Koch et al., 2003; Meiran, 2000). However, it is important to note that this finding cannot be attributed to the role of interference resolution in the current trial only. Rather, this finding might already represent (additional) carry-over effects because carry-over effects affect relevant and irrelevant secondary sequence switch trials differently. More precisely, persisting inhibition can never affect irrelevant secondary sequence switch trials because (by definition) the secondary sequence was never relevant in the given block and therefore could not have been attended in trial $n-1$. Therefore, the numerical difference in the size of switch costs between relevant and irrelevant secondary sequence trials is difficult to interpret.

More importantly, however, the present study demonstrates that after-effects of interference resolution influence the performance in switch trials and, thus, increase switch costs. Specifically, we found that both persisting activation of a previously relevant stimulus dimension as well as persisting inhibition of a previously irrelevant stimulus dimension led to an increased error rate in the current trial. Additionally, both findings appeared to be independent from each other. This suggests that a stimulus dimension that had to be attended in the previous trial is still activated and increases interference when it is present as the irrelevant dimension in the current trial (i.e., competitor priming). Similarly, a stimulus dimension that had to be inhibited in the previous trial appears to be still inhibited when being the relevant dimension in the current trial (i.e., negative priming).

Importantly, in previous studies, a differentiation between persisting activation and persisting inhibition was not possible because only two different stimulus dimensions were used (but see Waszak, Hommel, \& Allport,
2005, for item-specific effects of persisting activation and persisting inhibition). With only two stimulus dimensions, each task switch means a complete change of the relevant and irrelevant stimulus dimension and, therefore, is influenced by both persisting activation and persisting inhibition. In contrast, the design of the present experiment allowed us to disentangle these after-effects of interference resolution. Hence, the present data support theories assuming an effect of persisting activation of the relevant stimulus dimension (cf. Allport \& Wylie, 1999). Furthermore, the present data also provide evidence for the influence of persisting inhibition when switching between stimulus dimensions. In this context, one might speculate whether the finding of both effects and their apparent independence indicates that two different processes play a role in dimension switching: one focusing on the relevant stimulus dimensions, the other responsible for shielding the system from potentially distracting information (cf. Goschke, 2003).

The present findings were obtained using a SPT. Provided that switching effects are indicative of the cognitive representation of tasks, the present data show that attention to a stimulus dimension ("stimulus set", cf. Meiran, 2000) is a genuine part of a task representation. In previous studies, the SPT paradigm was found to activate the lateral premotor cortex (e.g., Schubotz \& von Cramon, 2001). A robust finding is that activation within this brain area depends on the attended stimulus dimension (e.g., color, form, or position). Yet, visual SPTs also overlap in a specific premotor subregion irrespective of the attended stimulus dimension (Schubotz \& von Cramon, 2003). Based on the assumption that interference arises from a sharing or crosstalk of brain areas, we can assume that the representational overlap in the lateral premotor cortex may suffice to cause interference between the attended stimulus dimensions and, thus, to result in task-switching phenomena like mixing costs and switch costs. In this context, our results suggest that the SPT provides an interesting paradigm to study switching between stimulus dimensions. However, further research is necessary to bring together anatomical differences in the representation of stimulus dimensions and empirical measures like mixing costs and switch costs.

Acknowledgments This research was funded by Deutsche Forschungsgemeinschaft Grant KO 2045/4-2 to Iring Koch and Grant SCHU 1439/1-2 to Ricarda I. Schubotz. We would like to thank Uta Zimmermann for conducting parts of the experiment. We would also like to thank Sander Los and Thomas Kleinsorge for helpful comments on earlier versions of this paper.

Open Access This article is distributed under the terms of the Creative Commons Attribution Noncommercial License which permits any noncommercial use, distribution, and reproduction in any medium, provided the original author(s) and source are credited. 


\section{References}

Allport, D. A., Styles, E. A., \& Hsieh, S. (1994). Shifting intentional set: Exploring the dynamic control of tasks. In C. Umiltà, \& M. Moscovitch (Eds.), Attention and Performance XV: Conscious and nonconscious information processing (pp. 421-452). Cambridge, MA: MIT Press.

Allport, A., \& Wylie, G. (1999). Task-switching: Positive and negative priming of task-set. In G. W. Humphreys, J. Duncan \& A. M. Treisman (Eds.), Attention, space and action: Studies in cognitive neuroscience (pp. 273-296). Oxford: Oxford University Press.

Braver, T. S., Reynolds, J. R., \& Donaldson, D. I. (2003). Neural mechanisms of transient and sustained cognitive control during task switching. Neuron, 39, 713-726.

Davidson, M. C., Amso, D., Anderson, L. C., \& Diamond, A. (2006). Development of cognitive control and executive functions from 4 to 13 years: evidence from manipulations of memory, inhibition, and task switching. Neuropsychologia, 44, 2037-2078.

Goffaux, P., Phillips, N. A., Sinai, M., \& Pushkar, D. (2006). Behavioural and electrophysiological measures of task switching during single and mixed-task conditions. Biological Psychology, 72, 278-290.

Goschke, T. (2003). Voluntary action and cognitive control from a cognitive neuroscience perspective. In S. Maasen, W. Prinz, \& G. Roth (Eds.), Voluntary action. An issue at the interface of nature and culture (pp. 49-85). Oxford: Oxford University Press.

Kleinsorge, T., Heuer, H., \& Schmidtke, V. (2004). Assembling a task space: global determination of local shift costs. Psychological Research, 68, 31-40.

Koch I., Prinz, W., \& Allport, A. (2005). Involuntary retrieval in alphabet-arithmetic tasks: Task-mixing and task-switching costs. Psychological Research, 69, 252-261.

Koch, I., Ruge, H., Brass, M., Rubin, O., Meiran, N., \& Prinz, W. (2003). Equivalence of cognitive processes in brain imaging and behavioral studies: evidence from task switching. Neuroimage, 20, 572-577.

Kray, J., \& Lindenberger, U. (2000). Adult age differences in task switching. Psychology \& Aging, 15, 126-147.

Los, S. A. (1996). On the origin of mixing costs: Exploring information processing in pure and mixed blocks of trials. Acta Psychologica, 94, 145-188.
Mayr, U. (2001). Age differences in the selection of mental sets: the role of inhibition, stimulus ambiguity, and response-set overlap. Psychology \& Aging, 16, 96-109.

Mayr, U., Diedrichsen, J., Ivry, R., \& Keele, S. W. (2006). Dissociating task-set selection from task-set inhibition in the prefrontal cortex. Journal of Cognitive Neuroscience, 18, 14-21.

Meiran, N. (2000). Reconfiguration of the stimulus task-set and the response task-set during task switching. In S. Monsell, \& J. Driver (Eds.), Attention \& performance XVIII: Control of cognitive processes (pp. 377-400). Cambridge, MA: MIT Press.

Meiran, N., \& Marciano, H. (2002). Limitations in advance task preparation: switching the relevant stimulus dimension in speeded same-different comparisons. Memory \& Cognition, 30, 540-550.

Monsell, S. (2003). Task switching. Trends in Cognitive Sciences, 7, 134-140.

Rogers, R. D., \& Monsell, S. (1995). Costs of a predictable switch between simple cognitive tasks. Journal of Experimental Psychology: General, 124, 207-231.

Rubin, O., \& Meiran, N. (2005). On the origins of the task mixing cost in the cuing task-switching paradigm. Journal of Experimental Psychology: Learning, Memory, \& Cognition, 31, 1477-1491.

Schubotz, R. I. (1999). Instruction differentiates the processing of temporal and spatial sequential patterns: evidence from slow wave activity in humans. Neuroscience Letters, 265, 1-4.

Schubotz, R. I., \& von Cramon, D. Y. (2001). Functional organization of the lateral premotor cortex: fMRI reveals different regions activated by anticipation of object properties, location and speed. Cognitive Brain Research, 11, 97-112.

Schubotz, R. I., \& von Cramon, D. Y. (2003). Functional-anatomical concepts of human premotor cortex: Evidence from fMRI and PET studies. Neuroimage, 20, 120-131.

Steinhauser, M., \& Hübner, R (2005). Mixing costs in task shifting reflect sequential processing stages in a multicomponent task. Memory \& Cognition, 33, 1484-1494.

Tipper, S. P. (2001). Does negative priming reflect inhibitory mechanisms? A review and integration of conflicting views. Quarterly Journal of Experimental Psychology, 54A, 321-343.

Waszak, F., Hommel, B., \& Allport, A. (2005). Interaction of task readiness and automatic retrieval in task switching: Negative priming and competitor priming. Memory \& Cognition, 33, 595610. 